\title{
MR Imaging Phenotypes and Features Associated with Pathogenic Mutation to Predict Recurrence or Metastasis in Breast Cancer
}

hong lu ( $\square$ honglu@tmu.edu.cn )

Tianjin Medical University Cancer Institute and Hospital

zhenzhen shao

Tianjin Medical University Cancer Institute and Hospital

jinpu yu

Tianjin Medical University Cancer Institute and Hospital

yanan cheng

Tianjin Medical University Cancer Institute and Hospital

wenjuan ma

Tianjin Medical University Cancer Institute and Hospital

peifang liu

Tianjin Medical University Cancer Institute and Hospital

\section{Research Article}

Keywords: Breast cancer, MRI phenotypes, biologic features, pathogenic mutation, disease recurrence or metastasis

Posted Date: February 4th, 2022

DOI: https://doi.org/10.21203/rs.3.rs-1290883/v1

License: @ (i) This work is licensed under a Creative Commons Attribution 4.0 International License. Read Full License 


\section{Abstract}

Objectives: To develop a multiple-feature model using clinico-pathological and radiologic characteristics adding pathogenic mutations associated signs for predicting recurrence or metastasis in breast cancers at high familial risk women.

Methods: Genetic testing for breast-related gene mutations was performed in 54 patients with breast cancers. Breast MRI findings were retrospectively evaluated in 64 tumors of 54 patients. The relationship between pathogenic mutation and clinico-pathological and radiologic features was examined, and disease recurrence or metastasis was estimated. Multiple logistic regression analyses were performed to identify independent factors of pathogenic mutation and disease recurrence or metastasis. Based on the above significant factors, a multivariate logistic regression was adopted to establish two models for predicting disease recurrence or metastasis in breast cancer by using $\mathrm{R}$ software.

Results: On the 64 breast cancers, the patients on 17 cancers have pathogenic mutation囚and no pathogenic mutation on 47 cancers. The clinico-pathogenic and radiologic features associated with pathogenic mutation included six signs: biologic features $(p=0.000)$, nuclear grade $(p=0.045)$, breast density $(p=0.005)$, MRI lesion type $(p=0.000)$, internal enhancement pattern $(p=0.004)$, and spiculated margin $(p=0.049)$. Necrosis within the tumors was only feature associated with increased disease recurrence or metastasis $(p=0.006)$. The model囚including clinico-pathologic and radiologic factors showed good discrimination in predicting disease recurrence or metastasis. The comprehensive model囚 including model『combined pathogenic mutations significantly associated signs is more effective.

Conclusions: The multiple-feature model in addition to pathogenic mutations associated parameters can more effective in predicting disease recurrence or metastasis.

\section{Key Points}

1) Only two MRI phenotypes - "internal enhancement patterns" and "central necrosis" were associated with disease recurrence or metastasis.

2) The MRI phenotypes associated with pathogenic mutation adding to the model for predicting disease recurrence or metastasis, the comprehensive model is an improvement based on multivariate analyses in breast cancer.

\section{Introduction}

Breast cancer is the most commonly diagnosed cancer, ranking fifth in the mortality of cancers in women in China ${ }^{[1]}$. Breast cancer risk is strongly influenced by genetic factors. The $B R C A 1$ and $B R C A 2$ are key genes associating with the development of breast cancer ${ }^{[2,3]}$. BRCA1 mutation carriers are more likely to have triplenegative and higher nuclear grade, which tend to have bad prognoses ${ }^{[4]}$. In addition, patients with metastasis or recurrence have also worse prognoses. Thus, many efforts to predict metastasis or recurrence in breast cancer patients, especially adding factors associated with pathogenic gene mutations have been made. 
Magnetic resonance imaging (MRI) has been widely used for screening women at increased risk for breast cancer ${ }^{[5]}$. The sensitivity of MRI for detecting breast tumors ranges from 77-91\%, which is higher than mammography (33\%-40\%), in women at high familial risk for breast cancer ${ }^{[6,7]}$. Breast MRI can both diagnose breast cancer accurately and predict prognosis using variable imaging features. Previous studies identified that peritumoral edema on T2-weighted images and higher degree of background parenchymal enhancement (BPE), and increased vascularity around the tumor, indicate poor prognosis in breast cancer ${ }^{[8-10]}$. Some studies have also demonstrated breast cancers with gene mutations had characteristic performances. The tumors with BRCA mutation carriers are associated with round shape, sharp margins, and rim enhancement

[11-13]. However, the current reported studies were little the relationship between pathogenic mutations and MRI features. In addition, multigene panel testing using NGS (next generation sequencing) technology could identify up to $50 \%$ more individuals with cancer susceptibility gene mutations in comparison with testing only for $B R C A 1$ and $B R C A 2^{[14]}$. Moreover, NGS technology has become possible to study a wider range of hereditary cancer related genes. The sequential analysis of genes has the disadvantage of been expensive, laborious and time consuming.

Therefore, the aim of the study was to retrospectively identify signs associated with pathogenic mutations in 16 genes, and furthermore to develop a multiple-feature model using clinico-pathological features and radiologic characteristics in addition to pathogenic mutations associated signs for predicting metastatic or recurrence in breast cancers at high familial risk women.

\section{Materials And Methods}

\section{Patients}

This retrospective study included patients with histologically confirmed invasive ductal carcinomas and ductal carcinomas in situ from July 2014 to January 2016. All patients had obtained informed consent. A total of 100 patients initially were included in study according to the following inclusion criteria: (a) preoperative MR images were acquired, (b) available pathological and immune-histochemical reports, (c) available test results for gene mutation status, (d) available clinical data, including age, familiar history of breast cancer, and TNM stage. Among them, 44 patients were excluded due to the following exclusion criteria: (a) patients confirmed benign lesions $(n=23)$, (b) patients received preoperative neoadjuvant chemotherapy treatment $(n=11),(c)$ patients received local resection of lesion before MRI examination $(n=10)$. Finally, 64 tumors of 56 patients were included, unilateral breast cancers on 48 patients, bilateral breast cancers on 6 patients, and two tumors of unilateral breast on 2 patients.

\section{Imaging Technique}

Dynamic breast MR imaging was performed using a $3.0 \mathrm{~T}$ magnet (GE Discovery 750) with patients in the prone position using a dedicated double-breast coil. Both breasts were imaged with axial T1-weighted sequence $(T R=622 \mathrm{~ms}, T E=10 \mathrm{~ms})$, fat-suppressed axial $T 2$-weighted sequence $(T R=6330 \mathrm{~ms}, T E=68 \mathrm{~ms})$, and axial DWI sequence $(T R=3235 \mathrm{~ms}, T E=64 \mathrm{~ms})$ with $b$ values of 0 and $1000 \mathrm{~s} / \mathrm{mm}^{2}$. The contrast enhancement scan was used sagittal T1-weighted fat-suppressed volume imaging for breast assessment 
(VIBRANT) sequence $\left(\mathrm{TR}=6.1 \mathrm{~ms}, \mathrm{TE}=2.9 \mathrm{~ms}\right.$, flip angle $=15^{\circ}$, matrix $=256 \times 128$, slice thickness $=1.8 \mathrm{~mm}$, phase acquisition time=90 100s), obtained before and continuous five times after intravenous injection of 0.1 $\mathrm{mmol} / \mathrm{L}$ gadolinium chelate per kilogram body weight (Gadovist, Bayer Schering Pharma, Berlin, Germany).

\section{Imaging Interpretation}

The MR imaging characteristics of tumors were described by using terminology according to BI-RADS [15]. Breast density included dense (heterogeneously dense and extremely dense) and non-dense (entinely fatty and scattered fibroglandular density). The lesion type was classified as mass, asymmetry/distortion, and calcification on mammogram and as mass and nonmass enhancement on MRI. The shape (regular or irregular), internal enhancement pattern (heterogeneous mass-enhancement, rim mass-enhancement, or line/segmental nonmass-enhancement), spiculated margin (yes or no), central necrosis within tumor (yes or no), peritumoral edema (yes or no)『and tumor localization (edge or central) were then evaluated on MRI.

\section{Histologic Evaluation and Pathogenic Mutation Analysis}

Histopathology, nuclear grade, tumor stage ( $T \backslash N \backslash M)$, and the status of immunohistochemical (IHC) staining for ER, PR, p53, Ki67, and HER2 were recorded, and positive for ER and PR was defined by the ASCO-CAP guideline [16]. Positive HER2 status was determined using IHC 3+ staining, or amplification using fluorescence in situ hybridization. Molecular subtypes were classified into Luminal A, Luminal B, HER2 over-expression, and Triple negative, and further classified into two groups including triple-negative (ER, PR, and HER negative) and non triple-negative. Biologic features were classified into three groups\unfavorable group (intermediate nuclear grade and ER negative/ PR negative, high nuclear grade and ER negative/ PR negative, high nuclear grade and ER negative/PR positive, high nuclear grade and ER positive/PR negative), favorable group (low nuclear grade and ER positive/ PR positive, low nuclear grade and ER negative/PR positive, low nuclear grade and ER positive/PR negative), and intermediate group (other nuclear grade and ER/PR). Disease recurrence or metastasis was defined as recurrence of breast cancer at any site (including local, regional, or distant).

Ion Ampliseq Designer (https://ampliseq.com/ Browse. action) was used to design the multiplex PCR amplification primers of the exon regions of 16 inherited breast cancer related genes

(BRCA1, BRCA2, CHEK2, PALB2, BRIP1, TP53, PTEN, STK11, CDH1, ATM, BARD1, MLH1, MRE11A, MSH2, MSH6, and $B A P 1$ ) with a coverage rate of $99.8 \%$, and Sanger sequencing was used to detect areas that could not be covered. According to the kit instructions, genomic DNA was extracted from the peripheral blood of breast cancer patients, and the DNA was amplified by multiplex PCR. The amplified products were connected, purified, oil-in-water reaction and enrichment of positive templates. Samples were sequenced in lon Proton using GPM 314 chip. After sequencing, Coverage Analysis and Variant Caller were downloaded, and IGV software was used to analyze the results and determine whether there were pathogenic mutation genes in the 16 genes.

\section{Statistical Analysis}


The chi-square or Fisher exact tests were used to compare proportions between two groups (pathogenic mutation vs. no pathogenic mutation and disease recurrence or metastasis vs. no disease recurrence or metastasis) by using SPSS software (version 20.0). Multiple logistic regression analyses were performed to identify independent factors that can be used to predict pathogenic mutation and disease recurrence or metastasis. Based on the above significant factors, a multivariate logistic regression was adopted to establish two models for predicting disease recurrence or metastasis in breast cancer by using $\mathrm{R}$ software (version 6.1, R Foundation for Statistical Computing, Vienna, Austria). Two-size $P \otimes 0.05$ indicated a significant difference. Model囚include clinico-pathologic features and radiologic variables, and Model囚include clinico-pathologic features and radiologic variables in addition to pathogenic mutations associated parameters. The performance was evaluated by area under the receiver operating characteristic (ROC) curve, accuracy, sensitivity, and specificity. The difference in the area under the curve (AUC) between Model『and Model $\square$ was analyzed by Delong's test.

\section{Results}

\section{Gene Mutations Status}

All 64 tumors of 56 patients (mean age, 43.5 years; age range, 26-72 years) were included. All patients have a family history of breast cancer. Of 64 tumors, gene mutations were detected in 28 tumors (43.8\%)(Fig.1), pathogenic mutations were identified in $17(26.6 \%)$ of 64 tumors, with 11 (17.2\%) carrying BRCA1 mutation, 2 (3.1\%) carrying BRCA2 mutation, and $4(6.3 \%)$ had been found pathogenic mutation in other genes including $\operatorname{ATM}(\mathrm{n}=1), B A P 1(\mathrm{n}=1), B R I P 1(\mathrm{n}=1)$, and $M S H 6(\mathrm{n}=1)$, respectively. Table 1 summarizes the 17 identified pathogenic mutations, which contain $10(58.8 \%)$ frameshift, 4 (23.5\%) nonsense, and $3(17.6 \%)$ splice-site variants in 17 breast cancers. No pathogenic mutations were identified in 11 of 64 tumors (17.2\%), with 4 carrying ATM mutation, 2 carrying MSH2 mutation, and BRIP1/BRCA1/PALB2/CDH1/TP53 had been found no pathogenic mutation on only one tumor, respectively. Table 2 summarizes the 11 identified no pathogenic mutations, which all were missense in 11 breast cancers. The other 36 tumors had no gene mutations in the 16 genes.

\section{Clinico-pathological and Radiologic Features associated with Pathogenic Mutations}

Associations between clinico-pathological factors and pathogenic mutation status are presented (Table 3 ). The women younger than 40-year-old with breast cancer have been more detected pathogenic gene mutation $(p=0.039)$. Pathogenic gene mutations subgroup had a significantly higher number of cancers with high nuclear grade ( 6 of 17 [35.3\%], $p=0.017$ ), ER negative (7 of 17 [41.2\%], $p=0.004)$, PR negative (7 of 17 [41.2\%], $p=0.007)$. No significant association was observed between histopathology, T stage, $\mathrm{N}$ stage, HER2 overexpression, p53 status and the pathogenic gene mutation. And there were almost significant difference in Ki67 status $(p=0.057)$ between pathogenic mutation and no pathogenic mutation. Pathogenic mutations were more likely to be the triple-negative phenotype (9 of 17 [52.9\%]) than other subtype (3 of 47 [6.4\%]) $(p=0.000)$. Significantly pathogenic mutation rates were observed in unfavorable biological behavior cancers (10 of 17 
[58.8\%], $p=0.000)$. Breast cancers carrying pathogenic mutations were more likely to being recurrence or metastasis (5 of 17 [29.4\%] and 3 of $47[6.4 \%])(p=0.014)$.

The mammographic and contrast-enhanced MR characteristics of the tumors in each genetic subgroup are presented in Table 4. On mammogram, dense breast in patients with pathogenic mutation (16 of 17 [94.1\%]) were significantly shower than those in patients with no pathogenic mutation (30 of $47[63.8 \%])(p=0.025)$. No mammographic lesion features were significantly associated with the pathogenic mutation status $(p=0.722)$. On MRI scan, cancers were identified as mass type in all 17 lesions $(100 \%)$ with pathogenic mutation, and as mass type in 37 lesions (78.7\%) and nonmass type in 10 lesions (21.3\%) with no pathogenic mutation $(p=0.038)$. We found difference in internal enhancement pattern $(p=0.000)$, spiculated margin $(p=0.005)$, or necrosis within tumor $(p=0.009)$ when comparing the pathogenic and non-pathogenic mutation subtypes. Breast cancers carrying pathogenic mutations showed more frequently ring mass enhancement with nonspiculated margin and necrosis within tumor. No significant associations were found between pathogenic mutation status with the shape $(p=0.253)$, edema around tumor $(p=0.847)$, or the location of the cancer $(p=0.623)$.

\section{Clinico-pathological and Radiologic Features associated with Disease Recurrence or Metastasis}

The 5-year rate of disease recurrence or metastasis was $12.5 \%$ (8/64) for all patients, $29.4 \%(5 / 17)$ for pathogenic mutation subgroup and $6.4 \%$ (3/47) for no pathogenic mutation subgroup, which was significant difference $(p=0.014)$. Tumors with five features including high nuclear grade (4 of $8[50.0 \%], p=0.004) \varangle \mathrm{ER}$ negative (5 of 8 [62.5\%], $p=0.030$ ), PR negative (5 of 8 [62.5\%], $p=0.041)$, p53 positive (7 of 8 [87.5\%], $p=0.046$ ) and triple negative subtype (4 of 8 [50.0\%], $p=0.015$ ) had more disease recurrence or metastasis during 5 years after surgery (Table 5). Only two radiologic features - "internal enhancement patterns" ( $p=0.045)$ and "central necrosis" ( $p=0.000$ ) were associated with disease recurrence or metastasis (Table 6). Breast cancers performing ring mass enhancement and central necrosis were more likely to being recurrence or metastasis.

\section{Multivariable Analyses of predicting Pathogenic Mutations and Disease Recurrence or metastasis and ROC Analyses}

These six features including biologic feature, nuclear grade, breast density, MRI lesion type, internal enhancement pattern and non-spiculated margin were proved to be independent prognostic factors predicting pathogenic mutations using multivariable logistic regression combined clinic-pathologic with radiologic features (Table 7). The AUC was 0.890 (95\%Cl: 0.793-0.988) (Fig.2), the sensitivity and specificity was 0.937 and 0.875 , respectively.

For predicting disease recurrence or metastasis, evaluating these seven clinico-pathologic and radiologic features including nuclear grade, ER, PR, p53, molecular subtype, internal enhancement pattern, and central necrosis, but the only one important and significantly independent prognostic factor was central necrosis within tumor as the Model $₫$ to predict disease recurrence or metastasis using multivariable logistic regression. 
The AUC was 0.839 (95\%Cl: 0.675-1.000) (Fig.3), the sensitivity and specificity was 0.963 and 0.600 ,

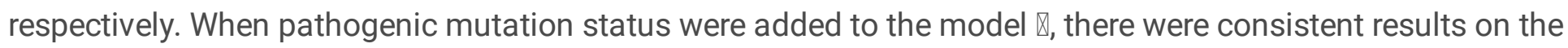
AUC, the sensitivity, and the specificity as the model $\nabla$.

For the Model $\nabla$ predicting disease recurrence or metastasis with the only one radiologic feature-"central necrosis within tumor" (Model $\varangle$ ) combined these six prognostic signs significantly associated with pathogenic mutations, the AUC was 0.857 (95\%Cl: 0.695-1.000) (Fig.3), the sensitivity and specificity was 0.964 and 0.750 , respectively. When clinico-pathologic and imaging features significantly associated with pathogenic mutations were added, the AUC increased from 0.839 to 0.857 , but had not significantly difference $(P=0.153)$, and the specificity increased from 0.600 to 0.750 (Fig.3).

\section{Discussion}

In our study, there were a $26.6 \%$ carrier rate of pathogenic mutation in the 64 breast cancers, consisting of $20.3 \%$ (13/64) in high-penetrance and 6.3\% (4/64) moderate/low- penetrance of the pathogenic mutation genes, and the prevalence of BRCA mutation was $20.3 \%$ (13/64). Tsaousis et al. ${ }^{[17]}$ showed that the pathogenic mutation frequencies of high-risk and moderate-risk genes was $16.5 \%$ and $6.2 \%$ respectively. Wang et al. ${ }^{[18]}$ detected a $13.5 \%$ carrier rate of pathogenic germline mutation in the 20 genes, but a $26.6 \%$ higher carrier rate of pathogenic mutation in this study. The underlying reasons may be that our enrolled women had high risk with family history of breast cancer.

Our results indicated that pathogenic mutation associated with younger than 40 years old, higher nuclear grade, triple-negative subtype, and unfavorable biological behavior, as well as dense breast on mammography. We also found that breast cancers with pathogenic mutations exhibited ring mass enhancement with nonspiculated margin on MRI. Although it was different from other studies focusing on the BRCA mutations that comparing the pathogenic mutation subgroup and the non-pathogenic mutation subgroup, 76.5\% (13/17) were $B R C A$ mutations in pathogenic mutations in this study. In agreement our observations, a significantly number of lesions described as rounded, with sharp margins, and with ring-enhancement was found to be higher in the BRCA mutation ${ }^{[13,19]}$. Yip et al. ${ }^{[20]}$ found that in the Asian population there is a significant association between triple negative and $B R C A 1$ but not BRCA2 status and a trend toward a higher percentage of grade 3 cancers in the BRCA1 carriers but not in the BRCA2 carriers. These above results were consistent to these characteristics observed in the current studies. In this study, we also found breast cancers with central necrosis were more frequently identified pathogenic mutation and disease recurrence or metastatic. The association of rim-enhancement with central necrosis or insufficient microvessel growth can be an indicator for the growth rate of tumors. Jimenez and coworkers have described centrally necrotizing carcinomas to have an accelerated clinical course and early systemic metastasis ${ }^{[21]}$. An accelerated growth rate can be associated with a high mitotic activity index (MAI) ${ }^{[19,22]}$. Several authors have implied that, due to the rapid growth rate of tumors in gene mutation carriers, the risk of recurrence or metastasis could high, so the screening frequency should be increased and $[23,24]$.

In this study, the 5-year rate of disease recurrence or metastasis was 12.5\% (8/64) for all patients, 29.4\% (5/17) for pathogenic mutation subgroup, and 6.4\% (3/47) for no pathogenic mutation subgroup. Wang et al. ${ }^{[18]}$ found that the rate of disease-free survival at 5 years was $73.3 \%$ among BRCA mutation carriers, as compared 
with $91.1 \%$ among non-carriers. Previous studies demonstrated that some factors including tumour size, multiple masses, fat-saturated $\mathrm{T}_{2} \mathrm{WI}$ signal, were confirmed to be associated with distant metastasis ${ }^{[25-26]}$. Some independent clinicopathologic factors were previously confirmed to be associated with distant metastasis. Age, T stage, N stage, lymphovascular invasion, and hormone receptor status were associated with bone metastasis in breast cancer ${ }^{[27]}$. The study found that sex, histological type, $N$ stage, grade, age, ER status, PR status can predict liver metastasis ${ }^{[28]}$. Lee et al. ${ }^{[29]}$ showed thatincreased ipsilateral vascularity and higher positive skewness of texture analysis were independently associated with disease recurrence, whereas rim enhancement showed no association with disease recurrence. In our study, central necorsis on MRI was the only factor associated with disease recurrence or metastasis, and the 5-year rate of disease-free survival was $50 \%(5 / 10)$ in the breast cancer with central necrosis, and 96.3\% (52/54) in the breast cancer without central necrosis. Although disease recurrence or metastasis differed among patients with nuclear grade, ER or PR status, p53 status, molecular subtype, and internal enhancement pattern were not confirmed to be a significant factors. The underlying reasons will be clarified with a larger sample size. Although adding the pathogenic mutation status could not improve the predicting efficiency, the six signs adding to the model for predicting disease recurrence or metastasis, the comprehensive model is an improvement of predictive models based on multivariate analyses. Spanberger et al. ${ }^{[30]}$ found that the extent of peritumoral edema on the preoperative T2weighted conventional MR scan showed to be related to the degree of angiogenesis, brain invasiveness, and overall survival. However, peritumoral edema is not a significant factor for predicting metastasis in this study, the possible reason maybe that there were brain metastases in only two patients(Fig. 4), and need to be further analyze with a larger sample size. Hence, using MRI as a screening tool enables the detection of breast cancer with pathogenic mutations in women with familial risk of breast cancer.

Our study had limitations. The sample size is very small which means that the differences or similarities regarding the clinical and pathological characteristics. Hence, these findings should to be confirmed by larger trials. Also, in this study all enrolled women had family history of breast cancer and were analyzed not only $B R C A$ mutation but also sixteen genes related breast cancer so it may be not reasonable to generalize these results to the entire population. Therefore, future studies may need broader inclusion criteria and enroll sporadic breast cancer cases from the general population. In addition, as the multigene panel testing is becoming widely adopted, studies should develop evidence-based practice guidelines.

In summary, clinico-pathologic and radiologic features of breast cancers can be effective in predicting pathogenic mutations and disease recurrence or metastasis, combined pathogenic mutations associated parameters enable more effective discrimination of disease recurrence or metastasis than use of clinicopathologic and radiologic variables alone.

\section{Abbreviations}

MRI:Magnetic resonance imaging;BPE:Background parenchymal enhancement;NGS:Next generation sequencing;TR:Time of repeatation;TE:Time of echo;DWI:Diffusion weighted imaging;VIBRANT:Volume imaging for breast assessment;BI-RADS:Breast imaging reporting and data system;IHC:Immunohistochemical;ER:Estrogen receptor;PR:Progesterone receptor;HER2:Human epidermal growth factor receptor-2;ASCO-CAP:American Society of Clinical Oncology-College of American 
Pathologists;PCR:Polymerase chain reaction;GPM:Global proteome machine;IGV:Integrative genomics viewer;ROC:Receiver operating characteristic;AUC:Area under the curve;MAl:Mitotic activity index.

\section{Declarations}

\section{Acknowledgements}

Not applicable.

\section{Authors' contributions}

ZZS, JPY,and HL designed the study. ZZS wrote the research. JPY analysed the expression of pathogenic gene mutations. YNC wrote the section of Pathogenic Mutation Analysis. WJM provided statistical advice for this manuscript. PFL reviewed the paper. All authors have read and approved the manuscript writing.

\section{Funding}

Grant: 1) Chinese National Key Research and Development Project [Grant number 2018YFC1315600]; 2) Tianjin health research project [Grant number KJ20024].

\section{Availability of data and materials}

The datasets used and/or analyzed during the current study available from the corresponding author on reasonable request.

\section{Ethics approval and consent to participate}

All procedures performed in this study involving human participants were in accordance with the Declaration of Helsinki (as revised in 2013). The study was approved by institutional ethics board of Tianjin medical university cancer institute and hospital (NO.: Ek2018125). Written informed consent was obtained from all subjects (patients) in this study.

\section{Consent for publication}

Not applicable.

\section{Competing interests}

All authors states that there are no potential competing interests. 


\section{Author details}

${ }^{1}$ Department of Breast Imaging, ${ }^{2}$ Cancer Molecular Diagnostics Core

Tianjin Medical University Cancer Institute and Hospital, National Clinical Research Center for Cancer, Key Laboratory of Breast Cancer Prevention and Therapy, Tianjin Medical University, Ministry of Education, Key Laboratory of Cancer Prevention and Therapy, Tianjin, P.R. China.

\section{References}

1. Lei $S$, Zheng $R$, Zhang $S$, et al. Breast cancer incidence and mortality in women in China: temporal trends and projections to 2030. Cancer Biol Med 2021;18: 470-476.

2. Miki Y, Swensen J, Shattuck-Eidens D, et al. A strong candidate for the breast and ovarian cancer susceptibility gene BRCA1. Science 1994; 266: 66-71.

3. Wooster R, Bignell G, Lancaster J, et al. Identification of the breast cancer susceptibility gene BRCA2. Nature 1995; 378:789-792.

4. Atchley DP, Albarracin CT, Lopez A, et al. Clinical and pathologic characteristics of patients with BRCApositive and BRCA-negative breast cancer. J Clin Oncol 2008; 26:4282-4288.

5. Kuhl C, Weigel S, Schrading S, et al. Prospective multicenter cohort study to refine management recommendations for women at elevated familial risk of breast cancer: the EVA trial. J Clin Oncol 2010; 28:1450-1457.

6. Kriege M, Brekelmans CT, Boetes C, et al. Efficacy of MRI and mammography for breast cancer screening in women with a familial or genetic predisposition. N Engl J Med 2004; 351:427-437.

7. Leach MO, Boggis CR, Dixon AK, et al. Screening with magnetic resonance imaging and mammography of a UK population at high familial risk of breast cancer: a prospective multicentre cohort study (MARIBS). Lancet 2005; 365:1769-1778.

8. Bae MS, Shin SU, Ryu HS, et al. Pretreatment MR Imaging Features of Triple-Negative Breast Cancer: Association with Response to Neoadjuvant Chemotherapy and Recurrence-Free Survival. Radiology 2016; 281: 392-400.

9. Hyejin, C, Kim HJ, Kim TH, et al. Invasive Breast Cancer: Prognostic Value of Peritumoral Edema Identified at Preoperative MR Imaging. Radiology 2018; 287: 68-75.

10. Lim Y, Ko ES, Han B, et al. Background parenchymal enhancement on breast MRI: association with recurrence-free survival in patients with newly diagnosed invasive breast cancer. Breast Cancer Res Treat 2017; 163:573-586.

11. Veltman J, Mann R, Kok T, et al. Breast tumor characteristics of BRCA1 and BRCA2 gene mutation carriers on MRI. Eur Radiol 2008; 18:931-938.

12. Noh JM, Han BK, Choi DH, et al. Association between BRCA Mutation Status, Pathological Findings, and Magnetic Resonance Imaging Features in Patients with Breast Cancer at Risk for the Mutation. J Breast Cancer 2013; 16: 308-314. 
13. Ha SM, Chae EY, Cha JH, et al. Association of BRCA Mutation Types, Imaging Features, and Pathologic Findings in Patients With Breast Cancer With BRCA1 and BRCA2 Mutations. AJR Am J Roentgenol 2017; 209: 920-928.

14. Desmond A, Kurian AW, Gabree M, et al. Clinical Actionability of multigene panel testing for hereditary breast and ovarian Cancer risk assessment. JAMA oncology 2015; 1:943-951.

15. American College of Radiology. ACR BI-RADS: magnetic resonance imaging. In: ACR Breast Imaging Reporting and Data System (BI-RADS) breast imaging atlas. Reston, VA: American College of Radiology, 2013.

16. Hammond ME, Hayes DF, Dowsett M, et al. American Society of Clinical Oncology/College of American Pathologists guideline recommendations for immunohistochemical testing of estrogen and progesterone receptors in breast cancer. Clin Oncol, 2010,28: 2784-2795.

17. Tsaousis GN, Papadopoulou E, Apessos A, et al. Analysis of hereditary cancer syndromes by using a panel of genes: novel and multiple pathogenic mutations. BMC Cancer 2019; 19:535.

18. Wang YA, et al. Germline breast cancer susceptibility gene mutations and breast cancer outcomes. BMC Cancer 2018; 18:315.

19. Veltman J, Mann R, Kok T, et al. Breast tumor characteristics of BRCA1 and BRCA2 gene mutation carriers on MRI. Eur Radiol 2008; 18:931-938.

20. Yip CH, Taib N.A., Choo W.Y., et al. Clinical and Pathologic Differences Between BRCA1-, BRCA2-, and NonBRCA-Associated Breast Cancers in a Multiracial Developing Country. World J Surg 2009; 33: $2077-2081$.

21. Jimenez RE, Wallis T, Visscher DW. Centrally necrotizing carcinomas of the breast: a distinct histologic subtype with aggressive clinical behavior. Am J Surg Pathol 2001; 25:331-337.

22. Tilanus-Linthorst M, Verhoog L, Obdeijn IM, et al. A BRCA1/2 mutation, high breast density and prominent pushing margins of a tumor independently contribute to a frequent false-negative mammography. Int $\mathrm{J}$ Cancer 2002; 102:91-95.

23. Kaas R, Kroger R, Hendriks JH, et al. The significance of circumscribed malignant mammographic masses in the surveillance of BRCA $1 / 2$ gene mutation carriers. Eur Radiol 2004; 14:1647-1653.

24. Komenaka IK, Ditkoff BA, Joseph KA, et al. The development of interval breast malignancies in patients with BRCA mutations. Cancer 2004; 100:2079-2083.

25. Ma W, Wang X, Xu Q, et al. Distant metastasis prediction via a multi-feature fusion model in breast cancer. Aging (Albany NY) 2020; 12:18151-18162.

26. Noda S, Onoda N, Asano Y, et al. T-stage and positive sentinel nodes ratio are the useful factors to predict non-sentinel node metastasis in breast cancer patients with macro-metastasis in the sentinel node. Int $\mathrm{J}$ Surg 2015; 14:56-60.

27. Delpech Y, Bashour SI, Lousquy R, et al. Clinical nomogram to predict bone-only metastasis in patients with early breast carcinoma. Br J Cancer 2015; 113:1003-1009.

28. Lin Z, Yan S, Zhang J, Pan Q. A nomogram for distinction and potential prediction of liver metastasis in breast cancer patients. J Cancer 2018; 9:2098-2106.

29. Lee J, Kim SH, Kang BJ. Prognostic Factors of Disease Recurrence in Breast Cancer Using Quantitative and Qualitative Magnetic Resonance Imaging (MRI) Parameters. Sci Rep 2020; 10:7598. 
30. Spanberger T, Berghoff AS, Dinhof C, et al. Extent of peritumoral brain edema correlates with prognosis, tumoral growth pattern, HIF1a expression and angiogenic activity in patients with single brain metastases. Clin Exp Metastasis 2013; 30:357-368.

\section{Tables}

Table 1

Pathogenic genes mutations in 15 patients with 17 cancers

\begin{tabular}{|c|c|c|c|c|}
\hline Gene & Mutation & Age at diagnosis & Family history & Mutation type \\
\hline \multirow[t]{9}{*}{ BRCA1 } & c.3359_3363delTTAAT & 48 & Sister & frameshift \\
\hline & c.5470_5477delATTGGGCA & 30 & Aunt & frameshift \\
\hline & c. $5136 \mathrm{G}>\mathrm{A}^{*}$ & 29 & Mother & nonsense \\
\hline & c. $5153-1 \mathrm{G}>\mathrm{A}^{*}$ & 47 & Sister & splice-site variants \\
\hline & chr17:41246509delG & 41 & Mother & frameshift \\
\hline & chr17:41226484insC & 44 & Sister & frameshift \\
\hline & c.5470_5477delATTGGGCA & 53 & Aunt & frameshift \\
\hline & chr17:41226455delG & 49 & Mother & frameshift \\
\hline & c. $2138 \mathrm{C}>\mathrm{G}$ & 34 & Mother, Aunt & nonsense \\
\hline \multirow[t]{2}{*}{ BRCA2 } & chr13:32913702DelTACT & 51 & Mother & frameshift \\
\hline & chr13:32914936InsTA & 41 & Sister & frameshift \\
\hline BAP1 & chr3:52440911InsC & 26 & Mother & frameshift \\
\hline$B R I P 1$ & chr17:59793425C>A & 41 & Sister & splice-site variants \\
\hline MSH6 & chr2:48010546delC & 49 & Mother & frameshift \\
\hline ATM & c. $2414 \mathrm{C}>\mathrm{T}$ & 32 & Aunt & nonsense \\
\hline
\end{tabular}

* indicate that the patients included two tumors. 
Table 2

No pathogenic genes mutations in 11 patients with 11 cancers

\begin{tabular}{lllll} 
Gene & Mutation & Age at diagnosis & Family history & Mutation type \\
\hline BRIP1 & chr17:59938867C>A & 75 & Sister & missense \\
\hline BRCA1 & c. $154 \mathrm{C}>\mathrm{T}$ & 46 & Sister & missense \\
\hline PALB2 & c. $3296 \mathrm{C}>\mathrm{T}$ & 43 & Sister & missense \\
\hline ATM & c. $8716 \mathrm{G}>\mathrm{C}$ & 47 & Sister & missense \\
\hline & c. $275 \mathrm{~A}>\mathrm{C}$ & 49 & Mother & missense \\
\hline & c. $125 \mathrm{~A}>\mathrm{G}$ & 43 & Aunt & missense \\
\hline c. $8495 \mathrm{G}>\mathrm{A}$ & 38 & Mother & missense \\
\hline CDH1 & c. $1888 \mathrm{C}>\mathrm{G}$ & 45 & Sister & missense \\
\hline MSH2 & c. $970 \mathrm{C}>\mathrm{T}$ & 42 & Mother & missense \\
\hline c. $14 \mathrm{C}>\mathrm{A}$ & 63 & 32 & Mother & missense \\
\hline c. $733 \mathrm{G}>\mathrm{A}$ & & Mother & missense
\end{tabular}


Table 3

Clinico-pathological characteristics of breast cancers carrying or not pathogenic mutations

Variable

Pathogenic No Pathogenic $\mathrm{N}=47$

$P$ value

$\mathrm{N}=17$

No. of all

Age

\begin{tabular}{llllr}
$\mathbb{8} 40$ years & $7(41.2 \%)$ & $7(14.9 \%)$ & $14(21.9 \%)$ & 0.039 \\
\cline { 1 - 2 }$\geq 40$ years & $10(58.8 \%)$ & $40(85.1 \%)$ & $50(78.1 \%)$ &
\end{tabular}

Histopathological type

\begin{tabular}{lllll} 
Ductal carcinoma in situ & $1(5.9 \%)$ & $7(14.9 \%)$ & $8(12.5 \%)$ & 0.434 \\
\cline { 1 - 2 } Invasive ductal carcinoma & $16(94.1 \%)$ & $40(85.1 \%)$ & $56(87.5 \%)$ & \\
\cline { 1 - 3 } Tumor size & & & & \\
\hline T1 & $8(47.1)$ & $15(31.9)$ & $23(35.9)$ & 0.534 \\
T2 & $7(41.2)$ & $26(55.3)$ & $33(51.6)$ \\
\hline T3 & $2(11.8)$ & $6(12.8)$ & $8(12.5)$
\end{tabular}

Node metastasis

\begin{tabular}{lllll} 
N- & $10(58.8)$ & $34(72.3)$ & $44(68.8)$ & 0.365 \\
\cline { 2 - 3 }$N+$ & $7(41.2)$ & $13(27.7)$ & $20(31.2)$ &
\end{tabular}

\section{Recurrence or Metastasis}

\begin{tabular}{|lllll} 
Yes & $5(29.4)$ & $3(6.4)$ & $8(12.5)$ & 0.014 \\
\cline { 1 - 3 } & $12(70.6)$ & $44(93.6)$ & $56(87.5)$ &
\end{tabular}

\section{Biologic feature}

\begin{tabular}{|c|c|c|c|c|}
\hline unfavorable & $10(58.8)$ & $6(12.8)$ & $16(25.0)$ & 0.000 \\
\hline Intermediate/ favorable & $7(41.2)$ & $41(87.2)$ & $48(75.0)$ & \\
\hline \multicolumn{5}{|l|}{ Nuclear grade } \\
\hline Low/Intermediate & $11(64.7)$ & $43(91.5)$ & $54(84.4)$ & \multirow[t]{2}{*}{0.017} \\
\hline high & $6(35.3)$ & $4(8.5)$ & $10(15.6)$ & \\
\hline \multicolumn{5}{|l|}{ ER } \\
\hline ER- & $10(58.8)$ & $9(19.1)$ & $19(29.7)$ & \multirow[t]{2}{*}{0.004} \\
\hline ER+ & $7(41.2)$ & 38 (80.9) & $45(70.3)$ & \\
\hline
\end{tabular}

PR 


\begin{tabular}{|c|c|c|c|c|}
\hline PR- & $10(58.8)$ & $10(21.3)$ & $20(31.2)$ & \multirow[t]{2}{*}{0.007} \\
\hline PR+ & $7(41.2)$ & 37 (78.7) & $44(68.8)$ & \\
\hline \multicolumn{5}{|l|}{ HER2 } \\
\hline HER2- & $13(76.5)$ & $29(61.7)$ & $42(65.6)$ & \multirow[t]{2}{*}{0.375} \\
\hline HER2+ & $4(23.5)$ & $18(38.3)$ & $22(34.4)$ & \\
\hline \multicolumn{5}{|l|}{ p53 } \\
\hline p53- & $5(29.4)$ & $24(51.1)$ & $29(45.3)$ & \multirow[t]{2}{*}{0.160} \\
\hline p53+ & $12(70.6)$ & 23 (48.9) & 35 (54.7) & \\
\hline \multicolumn{5}{|l|}{ Ki67 } \\
\hline Ki67- & $1(5.9)$ & $13(27.7)$ & $14(21.9)$ & \multirow[t]{2}{*}{0.057} \\
\hline Ki67+ & $16(94.1)$ & 34 (72.3) & $50(78.1)$ & \\
\hline \multicolumn{5}{|l|}{ Molecular substype } \\
\hline Luminal $\mathrm{A}$ & $3(17.6)$ & 15 (31.9) & $18(28.1)$ & \\
\hline Luminal B & $5(29.5)$ & $24(51.1)$ & $29(45.3)$ & \multirow[t]{2}{*}{0.000} \\
\hline HER2 over-expression & $0(0.0)$ & 5 (10.6) & $5(7.8)$ & \\
\hline Triple negative & $9(52.9)$ & $3(6.4)$ & $12(18.8)$ & \\
\hline \multicolumn{5}{|l|}{ Triple negative } \\
\hline No & $8(47.1)$ & $44(93.6)$ & $52(81.2)$ & \multirow[t]{2}{*}{0.000} \\
\hline Yes & $9(52.9)$ & $3(6.4)$ & 12 (18.8) & \\
\hline
\end{tabular}


Table 4

Radiologic Features of breast cancers carrying or not pathogenic mutations

Variable

Pathogenic No Pathogenic $\mathrm{N}=47$

$P$ value

$\mathrm{N}=17$

No. of all

MG

Breast density

Dense

Non-dense

1(5.9)

17(36.2)

$18(28.1) \quad 0.025$

Lesion type

Calcification

$3(17.6)$

$5(10.6)$

$8(12.5)$

0.722

Mass

$7(41.2)$

19 (40.4)

$26(40.6)$

Asymmetry/ Distortion

7(41.2)

$23(48.9)$

$30(46.9)$

MRI

Lesion type

Mass

17(100.0) $\quad 37(78.7)$

$54(84.4) \quad 0.038$

Nonmass

$0(0.0)$

$10(21.3)$

10 (15.6)

Internal enhancement pattern

Heterogeneous mass-enhancement

$4(23.5) \quad 30(63.8)$

$34(53.2) \quad 0.000$

Rim mass-enhancement

$13(76.5) \quad 7(14.9)$

20 (31.2)

Line/segmental nonmass-enhancement

$0(0.0 \%) \quad 10(21.3)$

$10(15.6)$

\section{Shape}

Regular

Irregular

$6(35.3)$

$10(21.3)$

$16(25.0)$

0.253

11 (64.7)

37 (78.7)

$48(75.0)$

\section{Spiculated margin}

Yes

$0(0.0)$

$16(34.0)$

$16(25.0) \quad 0.005$

No

17 (100.0)

$31(66.0)$

$48(75.0)$

\section{Central necrosis}

Yes

No

Peritumoral edema
$6(35.3)$

$11(64.7)$

$43(91.5)$
$10(15.6) \quad 0.009$

54 (84.4) 


\begin{tabular}{lcccc}
\hline Yes & $4(23.5)$ & $10(21.3)$ & $14(21.9)$ & 0.847 \\
\cline { 1 - 2 } No & $13(76.5)$ & $37(78.7)$ & $50(78.1)$ & \\
\hline Tumor localization & & & & \\
\hline Edge & $14(82.4)$ & $36(76.6)$ & $50(78.1)$ & 0.623 \\
\hline Central & $3(17.6)$ & $11(23.4)$ & $11(21.9)$ &
\end{tabular}




\section{Table 5}

Clinico-pathological characteristics of breast cancers with or not recurrence or metastasis

Variable

Recurrence or Metastasis

$\mathrm{N}=8$
No Recurrence or Metastasis $\mathrm{N}=56$

$P$ value

No. of

all

\section{Age}

$\llbracket 40$ years

$3(37.5)$

$11(19.6)$

14

0.253

(21.9)

$\geq 40$ years

$5(62.5)$

$45(80.4)$

50

(78.1)

Histopathological type

Ductal carcinoma in situ

1 (12.5)

7 (87.5)

$8(12.5) \quad 1.000$

Invasive ductal

carcinoma

7 (12.5)

49 (87.5)

56

(87.5)

Tumor size

$\mathrm{T} 1$

$2(25.0)$

$21(37.5)$

23

0.073

(35.9)

$\mathrm{T} 2$

$3(37.5)$

30 (53.6)

33

(51.6)

T3

$3(37.5)$

$5(8.9)$

8 (12.5)

\section{Node metastatic}

N-

$5(62.5)$

$\mathrm{N}+$

$3(37.5)$

Biologic feature

unfavorable

Intermediate/

favorable

Nuclear grade

Low/Intermediate

high

$4(50.0)$

$50(89.3)$

$6(10.7)$

12(21.4)

44(78.6)
39 (69.6)

44

(68.8)

17 (30.4)

20
16(25.0) $\quad 0.081$

48(75.0)
0.683

54

(84.4)

10(15.6)

ER 


\begin{tabular}{|c|c|c|c|c|}
\hline ER- & $5(62.5)$ & $14(25.0)$ & $\begin{array}{l}19 \\
(29.7)\end{array}$ & 0.030 \\
\hline ER+ & $3(37.5)$ & $42(75.0)$ & $\begin{array}{l}45 \\
(70.3)\end{array}$ & \\
\hline \multicolumn{5}{|l|}{ PR } \\
\hline PR- & $5(62.5)$ & $15(26.8)$ & $\begin{array}{l}20 \\
(31.2)\end{array}$ & \multirow[t]{2}{*}{0.041} \\
\hline PR+ & $3(37.5)$ & $41(73.2)$ & $\begin{array}{l}44 \\
(68.8)\end{array}$ & \\
\hline \multicolumn{5}{|l|}{ HER2 } \\
\hline HER2- & $7(87.5)$ & $35(62.5)$ & $\begin{array}{l}42 \\
(65.6)\end{array}$ & \multirow[t]{2}{*}{0.164} \\
\hline HER2+ & $1(12.5)$ & $21(37.5)$ & $\begin{array}{l}22 \\
(34.4)\end{array}$ & \\
\hline \multicolumn{5}{|l|}{ p53 } \\
\hline p53- & $1(12.5)$ & $28(50.0)$ & $\begin{array}{l}29 \\
(45.3)\end{array}$ & \multirow[t]{2}{*}{0.046} \\
\hline p53+ & $7(87.5)$ & $28(50.0)$ & $\begin{array}{l}35 \\
(54.7)\end{array}$ & \\
\hline \multicolumn{5}{|l|}{ Ki67 } \\
\hline Ki67- & $0(0.0)$ & $14(25.0)$ & $\begin{array}{l}14 \\
(21.9)\end{array}$ & \multirow[t]{2}{*}{0.110} \\
\hline Ki67+ & $8(100.0)$ & $42(75.0)$ & $\begin{array}{l}50 \\
(78.1)\end{array}$ & \\
\hline \multicolumn{5}{|l|}{ Molecular substype } \\
\hline Luminal A & $0(0.0)$ & $18(32.1)$ & $\begin{array}{l}18 \\
(28.1)\end{array}$ & \multirow[t]{4}{*}{0.052} \\
\hline Luminal B & $3(37.5)$ & $26(46.4)$ & $\begin{array}{l}29 \\
(45.3)\end{array}$ & \\
\hline HER2 over-expression & $1(12.5)$ & $4(7.2)$ & $5(7.8)$ & \\
\hline Triple negative & $4(50.0)$ & $8(14.3)$ & $\begin{array}{l}12 \\
(18.8)\end{array}$ & \\
\hline \multicolumn{5}{|l|}{ Triple negative } \\
\hline No & $4(50.0)$ & $48(85.7)$ & $\begin{array}{l}52 \\
(81.2)\end{array}$ & \multirow[t]{2}{*}{0.015} \\
\hline Yes & $4(50.0)$ & $8(14.3)$ & $\begin{array}{l}12 \\
(18.8)\end{array}$ & \\
\hline
\end{tabular}


Table 6

Radiologic Features of breast cancers with or not recurrence or metastasis

Variable

Recurrence or

Metastasis $\mathrm{N}=8$

No Recurrence or

Metastasis $\mathrm{N}=56$

$P$ value

No. of

all

MG

\section{Breast density}

\begin{tabular}{|c|c|c|c|c|}
\hline Dense & $1(12.5)$ & $17(30.4)$ & $18(28.1)$ & 0.293 \\
\hline Non-dense & $7(87.5)$ & $39(69.6)$ & $46(71.9)$ & \\
\hline \multicolumn{5}{|l|}{ Lesion type } \\
\hline Calcification & $1(12.5)$ & $7(12.5)$ & $8(12.5)$ & \multirow[t]{3}{*}{0.831} \\
\hline Mass & $3(37.5)$ & $22(39.3)$ & $\begin{array}{l}26 \\
(40.6)\end{array}$ & \\
\hline Asymmetry/ Distortion & $4(50.0)$ & $27(48.2)$ & $\begin{array}{l}30 \\
(46.9)\end{array}$ & \\
\hline
\end{tabular}

\section{MRI}

Lesion type

Mass

$6(75.0)$

48 (85.7)

54

(84.4)

Nonmass

2(25.0)

$8(14.3)$

10

(15.6)

Internal enhancement pattern

\begin{tabular}{lccll}
$\begin{array}{l}\text { Heterogeneous mass- } \\
\text { enhancement }\end{array}$ & $1(12.5)$ & $33(58.9)$ & $\begin{array}{l}34 \\
(53.1)\end{array}$ & $\mathbf{0 . 0 4 5}$ \\
\hline Rim mass-enhancement & $5(62.5)$ & $15(26.8)$ & $\begin{array}{l}20 \\
(31.2)\end{array}$ \\
\hline $\begin{array}{l}\text { Line/segmental nonmass- } \\
\text { enhancement }\end{array}$ & $2(25.0)$ & $8(14.3)$ & $\begin{array}{l}10 \\
(15.6)\end{array}$ \\
\hline Shape & & & & 0.081 \\
Regular & $4(50.0)$ & $12(21.4)$ & $\begin{array}{l}16 \\
(25.0)\end{array}$ & $\begin{array}{l}48 \\
(75.0)\end{array}$
\end{tabular}

\section{Spiculated margin}

Yes

$0(0.0)$

$16(28.6)$

16

0.081 
(25.0)

No

$8(100.0)$

$40(71.4)$

48

(75.0)

\section{Central necrosis}

\begin{tabular}{llll} 
Yes & $6(75.0)$ & $4(7.1)$ & \multicolumn{1}{c}{$\begin{array}{l}10 \\
\text { (15.6) }\end{array}$} \\
\hline No & $2(25.0)$ & $52(92.9)$ & 54 \\
\end{tabular}

\section{Peritumoral edema}

\begin{tabular}{|c|c|c|c|c|}
\hline Yes & 3 (37.5) & $11(19.6)$ & $\begin{array}{l}14 \\
(21.9)\end{array}$ & 0.253 \\
\hline No & $5(62.5)$ & 45 (80.4) & $\begin{array}{l}50 \\
(78.1)\end{array}$ & \\
\hline \multicolumn{5}{|c|}{ Tumor localization } \\
\hline Edge & $6(75.0)$ & 44 (78.6) & $\begin{array}{l}50 \\
(78.1)\end{array}$ & 0.819 \\
\hline Central & $2(25.0)$ & $12(21.4)$ & $\begin{array}{l}14 \\
(21.9)\end{array}$ & \\
\hline
\end{tabular}




\section{Table 7}

Multiple logistic regression analysis predicting pathogenic mutation and recurrence or metastasis on breast cancers

Variable

\section{Pathogenic mutations}

Age

$₫ 40$ years

$\geq 40$ years

\section{Recurrence or Metastasis}

No

Yes

\section{Biologic feature}

unfavorable

Intermediate/ favorable

Nuclear grade

Low/Intermediate

high

ER

ER+

ER-

PR

PR+

PR-

Molecular substype

Non-Triple negative

Triple negative

\section{Breast density}

Non-dense

Dense

MRI Lesion type

Nonmass
Odds ratio

$95 \% \mathrm{Cl}$

$P$ value

Reference

NA

0.418

0.914

$0.736-1.134$

Reference

NA

0.039

1.402

1.024-1.916

Reference

NA

0.000

0.409

0.259-0.647

Reference

NA

0.045

0.736

0.551-0.986

Reference

NA

0.416

1.368

0.646-2.896

Reference

NA

0.304

1.399

$0.742-2.642$

Reference

NA

0.634

1.099

0.721-1.676

Reference

NA

0.005

1.315

1.093-1.582

Reference

NA

0.000 


\begin{tabular}{|c|c|c|c|}
\hline Mass & 2.705 & $1.739-4.207$ & \\
\hline \multicolumn{4}{|c|}{ Internal enhancement pattern } \\
\hline Other enhancement types & Reference & NA & \multirow[t]{2}{*}{0.004} \\
\hline Rim mass-enhancement & 1.404 & $1.121-1.758$ & \\
\hline \multicolumn{4}{|l|}{ Spiculated margin } \\
\hline Yes & Reference & NA & \multirow[t]{2}{*}{0.049} \\
\hline No & 0.803 & $0.649-0.994$ & \\
\hline \multicolumn{4}{|l|}{ Central necrosis } \\
\hline No & Reference & NA & \multirow[t]{2}{*}{0.388} \\
\hline Yes & 0.875 & $0.649-1.181$ & \\
\hline
\end{tabular}

\section{Recurrence or metastasis}

Nuclear grade

\begin{tabular}{llll} 
Low/Intermediate & Reference & NA & 0.056 \\
\cline { 1 - 2 } high & 1.242 & $0.998-1.544$
\end{tabular}

\section{ER}

$\begin{array}{llll}\text { ER+ } & \text { Reference } & \text { NA } & 0.888 \\ \text { ER- } & 1.042 & 0.590-1.839 & \end{array}$

PR

$\begin{array}{llll}\text { PR+ } & \text { Reference } & \text { NA } & 0.920 \\ \text { PR- } & 0.973 & 0.573-1.653 & \end{array}$

p53

p53-

Reference

NA

0.348

p53+

1.069

$0.930-1.230$

Molecular subtype

Non-Triple negative

Reference

NA

0.498

Triple negative

0.909

$0.691-1.196$

\section{Internal enhancement pattern}

Other enhancement types

Reference

NA

0.439

Rim mass-enhancement

1.037

$0.946-1.138$

Central necrosis

No

Reference

NA 
Figures
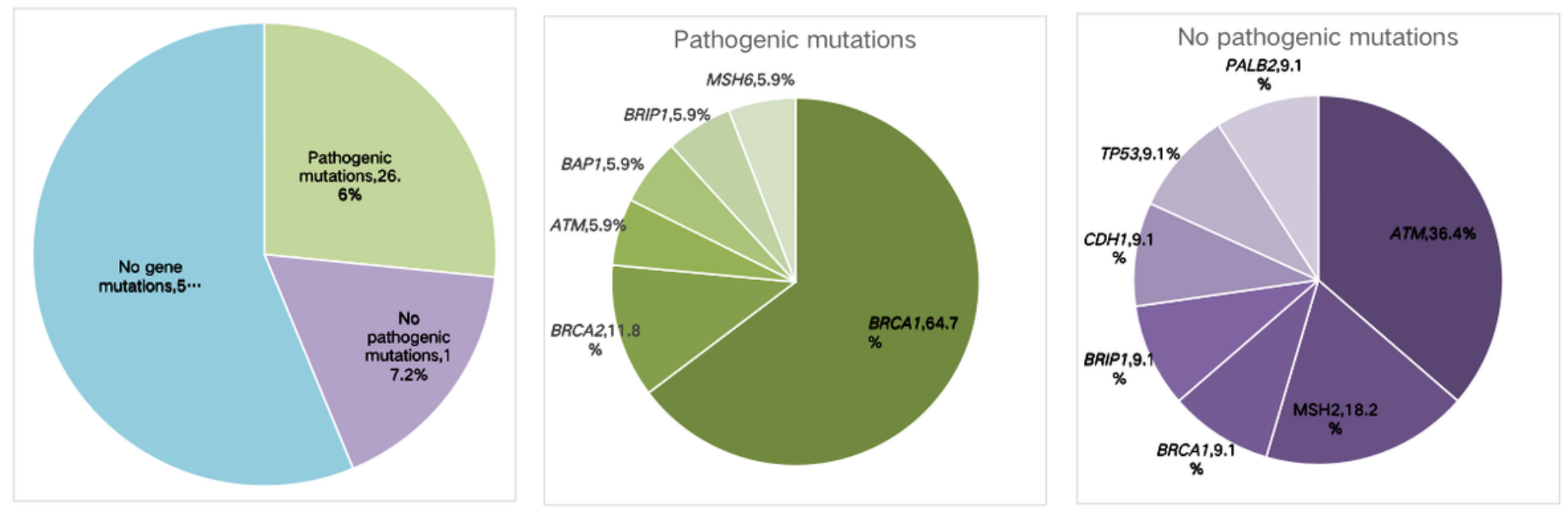

Figure 1

Distribution of the 16 susceptibility mutations in 64 breast carcinomas. Genes not shown (PTEN, STK11, CHEK2, BARD1, MLH1, MRE11A) are those without identified mutations in the study cohort.

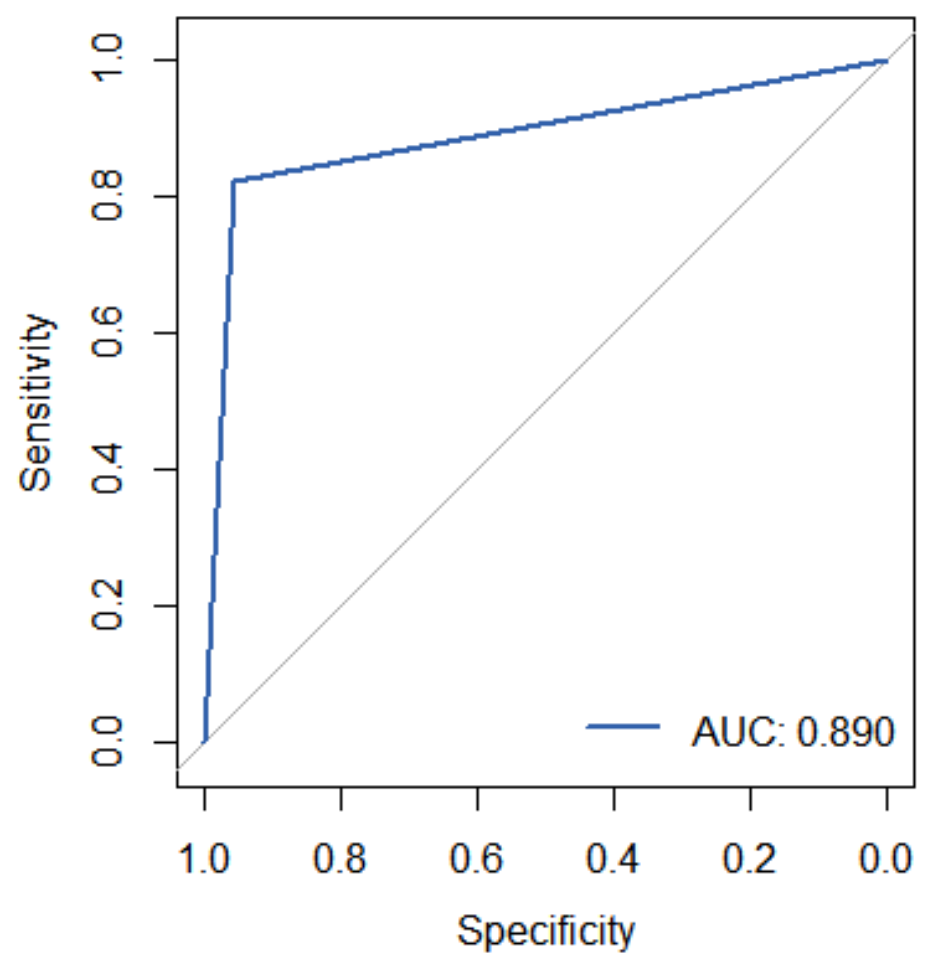

Figure 2

Receiver operating characteristic curves used to predict pathogenic mutation with clinico-pathologic and radiologic features 


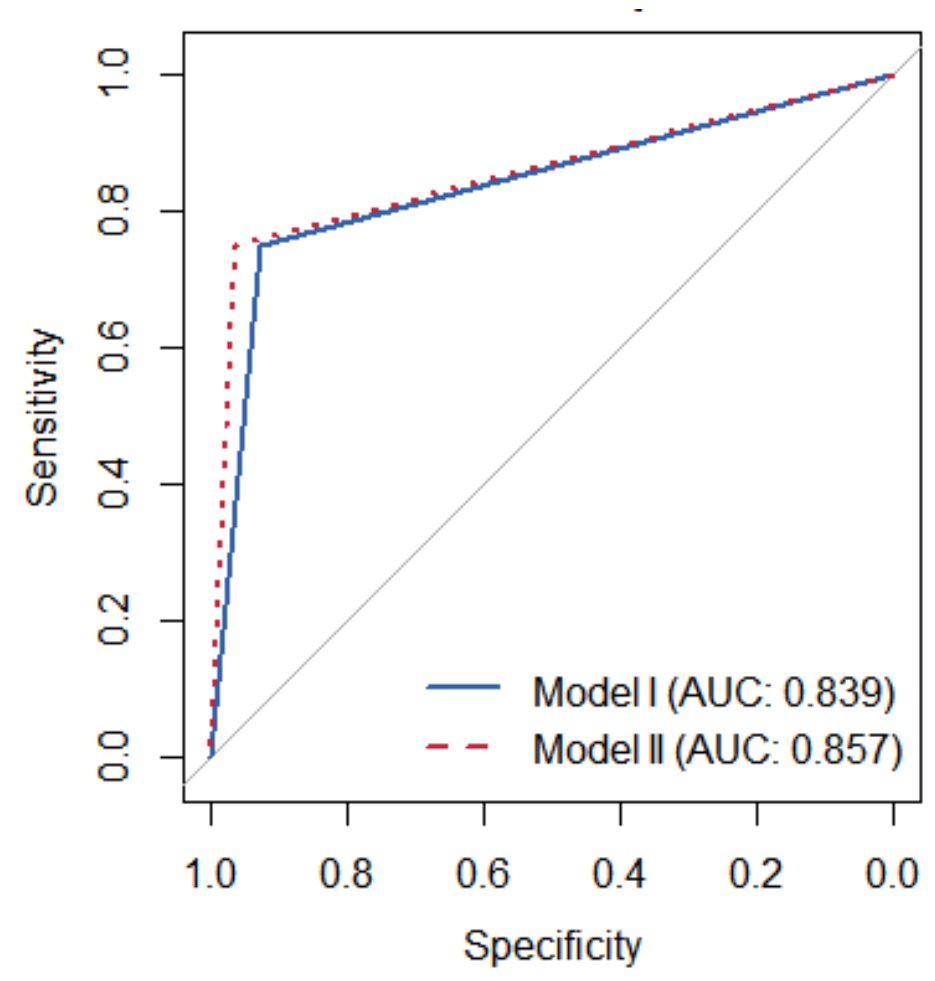

Figure 3

Receiver operating characteristic curves used to predict recurrence or metastasis with clinico-pathologic and radiologic features alone (Model $\varangle$ ) and combined with signs significantly associated with pathogenic mutation (Model囚)

\section{Figure 4}

A 29-year-old woman with BRCA1 mutation. (A) An axial fat-suppressed T2-weighted magnetic resonance image demonstrated central necrosis. (B) An axial contrast-enhanced T1-weighted magnetic resonance image demonstrated rim enhancement. ( $C$ and $D$ ) This patient experienced brain metastasis after 5 years from the date of surgery. Axial contrast-enhanced T1-weighted images demonstrated multiple lesions showing rim enhancement. 